\title{
Wetland vegetation of Biratnagar, Nepal
}

\author{
Bhabindra Niroula and Sasinath Jha \\ Department of Botany, Post Graduate Campus, Tribhuvan University \\ Biratnagar, Nepal \\ E-mail: niroulab@gmail.com
}

\begin{abstract}
In total, 128 species of aquatic macrophytes (angiosperms 120, pteridophytes 6 , bryophyte 1 and alga 1) were recorded among which 37 species were helophytes, 35 tenagophytes, 37 hyperhydates, 6 epihydates, 5 vittates, 1 rosulate and 5 pleustophytes on the basis of growth form. Based on the frequency of occurrence $2 \%$ were abundant, $12 \%$ frequent, $76 \%$ occasional and $10 \%$ scarce. Seven macrophyte communities were recorded on the basis of habitat. These were: 1. deep water submerged associes; 2 . shallow water submerged associes; 3. Floating- leaf form associes; 4. free floating-leaf form associes; 5. Amphibious-marsh associes; 6. moist meadow associes; and 7. dry mud associes.
\end{abstract}

Key words: Aquatic macrophytes, growth forms, use potential

\section{Introduction}

Wetlands have high ecological, socio-economic and cultural significance (Mitsch and Gosselink 2000). They harbor different species of flora and fauna and many ethnic groups are dependent on wetlands for their livelihoods. Nepal's wetlands are continuously being lost to agriculture, human settlement and urbanization as well as from water pollution by domestic sewage, industrial effluents and agricultural runoffs. Growing environmental pollution has caused eutrophication of many ponds, lakes and rivers (GON, 2014). These are the most threatened ecosystems and are disappearing at an alarming rate because of poor understanding of their values (IUCN/ Nepal 1992). As the wetland vegetation is the essential components of aquatic ecosystems, status and wealth of information is inevitable for wetland restoration.

Biratnagar township (lat N $26^{0} 20^{\prime}$, long E $87^{0} 16^{\prime}$; alt $72 \mathrm{~m} \mathrm{msl}$ ) is a part of the Indo-Gangetic flood plain. It has alluvial soil and tropical monsoonic climate with average annual rainfall 1348 $\mathrm{mm}$ and average annual air temperature $22{ }^{\circ} \mathrm{C}$. It is bordered by Keshaliya river in the west and Singhia river in the east and possesses several derelict depressions usually inundated during rainy season. Besides these perennial water bodies it has numerous seasonal wetlands such as irrigational canals, ditches along the roadsides, manmade ponds and deep water paddy fields. The present study aims to highlight the species composition, growth forms, communities, and human use potential of wetland macrophytic flora of Biratnagar township.

\section{Materials and Methods}

Wetland vegetation of Biratnagar was observed for two years from January 2011 to December 2012. The specimens of the aquatic macrophytes were collected manually at monthly intervals. They were recorded, tagged and pressed to prepare herbaria. The plants were identified as per Hooker (1872-1897), Cook (1996) and Siwakoti and Varma (1999). The identified specimens were confirmed by making crosschecks with the specimens housed at the herbarium Centre, University Department of Botany, T.M. Bhagalpur University, Bhagalpur, India. The specimens are deposited at Regional Herbarium Centre, Post Graduate Campus, T.U., Biratnagar, Nepal. 
The nomenclature of plants is based on Hara et al. (1978-1982) and Press et al. (2000). Growth form categories of the plants were determined as per Cook (1996). The frequency of occurrence of each species was recorded as abundant (++++), frequent $(+++)$, occasional $(++)$ and scarce $(+)$ on the visual basis. Uses of the plants were determined either through interviews with local people or with the help of standard literature (Anonymous 1948-1976, Banerjee 1995, Bala and Mukherjee, 2007, GON 2007). Growth form categories of the plants were determined as per Cook (1996) as follows:

A. Plants not physiologically bound to water but tolerating longer periods of submergence: Helophytes

B. Plants physiologically bound to water at least part of the generative cycle:

1. Plants with the juvenile phase submerged in or floating on water and the adult phase (flowering) phase terrestrial: Tenagophytes

2. Plants rooted in the substrate with all photosynthetic parts submerged:

(a) Leaves borne in rosette: Rosulate

(b) Leaves arranged along elongated stems: Vittates

3. Plants with photosynthetic parts in contact with air:

(a) Plants free floating on the surface, not attached to or penetrating the substrate: Pleustophytes

(b) (b) Plants with roots penetrating the substrate:

(i) Leaves and/or stems floating on but not arising above water surface: Epihydates

(ii) Leaves and / or stems emerging above the water surface: Hyperhydates

\section{Results and Discussion}

Species composition: The aquatic macrophytes showed diversity in floristic composition. Altogether 128 species of aquatic macrophytes with 92 genera belonging to 45 families were recorded from the wetlands of Biratnagar township. They comprised 120 species of angiosperms (dicots 54 and monocots 66); pteridophyte 6, bryophyte 1 and macroalga 1 (Table 1). Among the families of angiosperms, the dominant ones in number of species included: poaceae (27), Cyperaceae (17), Asteraceae (9), Polygonaceae (6), Lythraceae and Scrophulariaceae (4), amaranthaceae, Apiaceae, Araceae, Commelinaceae and Pontederiaceae (3). The dominance of grasses and sedges corresponded with the findings of Dangol et al. (1986), Shrestha (1996) and Niroula and Singh (2011). The dominance of monocots over dicots by species count but reverse case by number of families approached the study of Satyanarayan (1962) Shrestha (1996), and Niroula and Singh (2011). Seventy-six percent plant species were occasional, $12 \%$ frequent, $10 \%$ scarce and $2 \%$ abundant in occurrence. Colocasia esculenta, Eichhornia crassipes and Oryza sativa were abundant while Aeschynemone asper, Ammania auriculata, Cyperus pilosus, Leptochloa chinensis, Schoenoplectus juncoides, Smithia ciliata and Utricularia exoleta were scarce aquatic macrophytes.

Table 1. Taxonomic composition of wetland macrophytes.

\begin{tabular}{lcccccc}
\hline Taxa & Macroalgae & Bryophyta & Pteridophyta & Dicotyledonae & Monocotyledonae & Total \\
\hline Families & 1 & 1 & 6 & 23 & 14 & 45 \\
Genera & 1 & 1 & 6 & 39 & 45 & 92 \\
Species & 1 & 1 & 6 & 54 & 66 & 128 \\
\hline
\end{tabular}

Growth forms: Seven growth forms of aquatic macrophytes were recorded in wetland areas of Biratnagar township. Number of species in each growth forms were: helophytes (37) = hyperhydates (37) > tenagophytes (35) > epihydates (7) > vittates (6) > pleustophytes (5) > 
rosulates (1). Emergents (helophytes, tenagophytes, and hyperhydates) had the highest contribution in comparison to submerged (vittates, rosulates), rooted floating - leaved (epihydates), and free - floating (pleustophytes) species. Percentage contribution of seven growth forms of macrophytic vegetation at Biratnagar township is shown in figure 1. Upadhyay et al. 2011 recorded four growth form categories: emergent (13), floating leaved (6), free floating (5) and submerged (4) from the selected wetland habitats of Biratnagar township. In the present work there was also a general decreasing trend in diversity of aquatic macrophytes from shore line to the centre of the water bodies. The dominance of emergents can be attributed to the edge effect of ecotone (Odum, 1971).

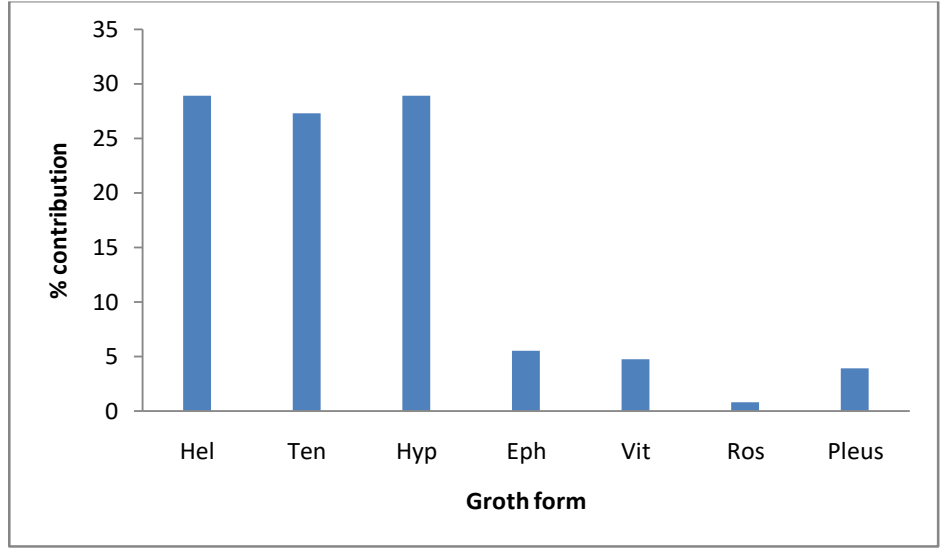

Figure 1. Growth forms distribution of wetland vegetation in the study area.

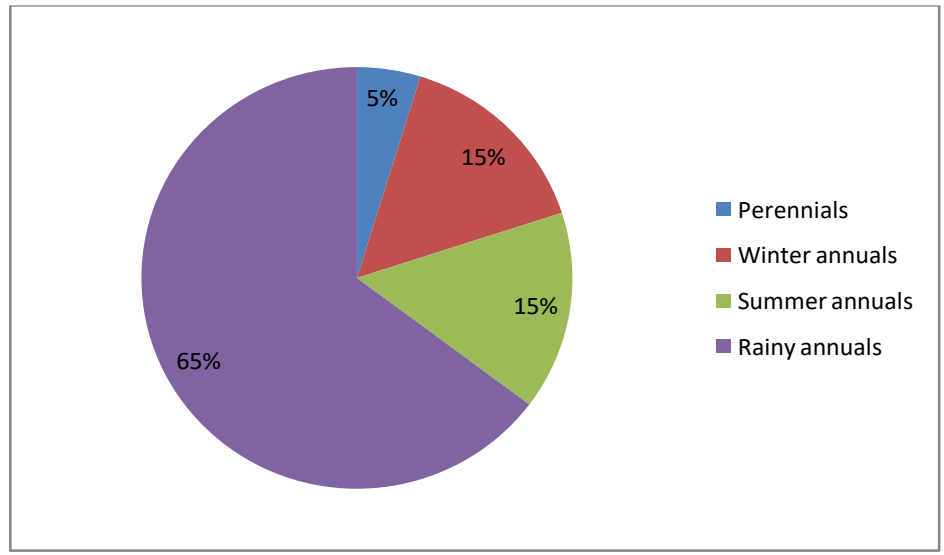

Figure 2. Habits of aquatic macrophytes in the study area.

Seasonal distribution: There was seasonal variation in the occurrence of different growth forms and species composition. Helophytes, tenagophytes and hyperhydates (mostly grasses and sedges); epihydates and rosulates were dominant in the rainy season. Most pleustophytes (free floating) Azolla imbricata, Lemna aequinoctialis, Spirodella polyrhiza and Pistia stratioites); few tenagophytes (Hygrophila polysperma, Rumex dentatus, Sagittaria trifolia and Veronica anagallis-aquatica); 
hyperhydate (Ranunculus scleratus) and vittates (Chara schweinitzii, Utricularia spp.) were the common winter species. Dominant aquatic macrophytes of summer season were: Alternanthera philoxeroides, Marsilea minuta, Monochoria hastata, Oenanthe javanica, Panicum psilopodium, Sacciolepis interrupta (hyperhydates); Colocasia esculenta, Hemarthra compressa, Polygonum barbatum, Spilanthes iabadicensis (tenagophytes) and Potamogeton crispus (vittate). Acorus calamus, Arundinella benghalensis, Canna indica, Colocasia esculenta, Crinum asiaticum, Saccharum spontaneum, Typha angustifolia, T. elephantina, Vetiveria zizanoides were perennial aquatic macrophytes. Percentage occurrence of winter, summer, rainy annuals and perennial aquatic macrophytes are given in fig 2.

Association of macrophytic communities: Wetland vegetation was characterized into following communities as per their habitat conditions.

1. Deep water submerged associes (vittates): Hydrilla verticillata, Ottelia alismoides.

Habitat: Depth and turbidity of water affecting the penetration of light, a silty substratum and little biotic disturbance.

2. Shallow water submerged associes (vittates): Chara schweinitzii, Potamogeton crispus. Habitat: a hard bottomed clayey/silty substratum with transparent shallow water depth.

3. Floating - leaf form associes (epihydates): Nymphaea pubescens, Sagittaria guyanensis, Ipomoea aquatica.

Habitat: minimum biotic disturbance, very gradual change in the level of water and a substratum of loose mud.

4. Free floating - leaf form associes (pleustophytes): Azolla imbricata, Lemna minor, Spirodela polyrhiza, Pistia stratiotes, Eichhornia crassipes.

Habitat: minimum water disturbances, shallow to deep stagnant water at winter season and substratum of a loose mud.

5. Amphibious - marsh associes (helophytes, tenagophytes and hyperhydates):

(a) Typha angustifolia and Cyperus spp. in the permanent clayey/silty bottomed permanent waters.

(b) Alternanthera philoxeroides, A. sessilis, Echinocloa colona, Marsilea minuta in the temporary waters.

(c) Hygrophila auriculata, Eleocharis acutangula, Cyperus esculentus, Fimbristylis miliacea etc. on a clayey substratum.

(d) Paspalidium punctatum, Sacciolepis interrupta, Ludwigia perennis, Arundinella bengalensis, Polygonum spp. inundated for a long period and a substratum of an organic brown mud and stiff clay.

(e) Ranunculus scleratus, Rumex dentatus sometimes Veronica anagallis-aquatica, loose bottomed organic clayey soil with gradual decreasing water levels.

6. Moist meadow associes (helophytes, tenagophytes): Cynodon dactylon, Panicum psilopodium, Paspalum scrobiculatum, Cyperus iria.

Habitat: gradually slopping moist substratum.

7. Dry mud associes (helophytes): Polygonum plebeium, Grangea maderaspatana, Sphaeranthus indicus, Cotula hemisphaerica, Gnaphalium polycaulon.

Habitat: drying clay, moderate grazing.

Macrophytic communities with their associes in the wetlands of Biratnagar were in agreement with Misra (1976) in the Varanasi on the bank of river Ganga.

Uses of aquatic plants: Aquatic macrophytes were observed to be useful in the local socioeconomy, religion, culture, and ecology. Use category of wetland plants occurring at Biratnagar township is given in Table 2. They were observed to play substantial roles are as: feed for livestock (41 species), edible/pot herb (23 species), fish poison (2 species), green manure/compost (10 species), genetic resource breeding stock (3 species), handicrafts/thatch and cordage (9 species), insecticides (3 species), medicinal (30 species), and religious (5 
species). Other uses included: dyes-Eclipta prostrata, Polygonum hydropiper; aquarium plantCeratophyllum demersum, Hydrilla verticillata; spawning/nesting habitats and feed for aquatic invertebrates/waterfowls - Nymphaea pubescens, Nymphoides spp., Potamogeton crispus, Saccharum spontaneum; fire wood/hedge plant-Ipomoea carnea.

In genral, native aquatic flora is being outcompeted by invasive alien species such as $E$. crassipes, A. philoxeroides, I carnea ssp. fistulosa, M. micrantha and P. stratiotes. On the other hand, wetlands are disappearing at an alarming rate due to unplanned development of the township.

Table 2. List of aquatic macrophytes of Biratnagar with growth forms, frequency of occurrence

\begin{tabular}{|c|c|c|c|c|}
\hline $\mathrm{S}, \mathrm{N}$ & Growth form/species & Family & Freq. & Uses \\
\hline & Helophytes & & & \\
\hline 1. & Alternanthera paronychioides St. Hil & Amaranthaceae & ++ & - \\
\hline 2. & A.sessilis (L.) DC. & Amaranthaceae & ++ & $\mathrm{F}$ \\
\hline 3. & Ammania baccifera $\mathrm{L}$. & Lythraceae & ++ & - \\
\hline 4. & Caesulia axillaris Roxb. & Asteraceae & ++ & M \\
\hline 5. & Centella asiatica (L.) Urb. & Apiaceae & ++ & M \\
\hline 6. & Coix lacryma-jobi $\mathrm{L}$. & Poaceae & + & M \\
\hline 7. & Commelina benghalensis L. & Commelinaceae & +++ & M \\
\hline 8. & C. paludosa Blume & Commelinaceae & + & $\mathrm{F}$ \\
\hline \multirow[t]{2}{*}{9.} & Cotula hemispherica (Roxb.) Wall. ex C. & & & \\
\hline & B. Clarke & Asteraceae & ++ & $\mathrm{C}$ \\
\hline 10. & Crinum asiaticum $\mathrm{L}$. & Amarylidaceae & ++ & - \\
\hline 11. & Cynodon dactylon (L.) Pers. & Poaceae & +++ & $\mathrm{M} / \mathrm{FO}$ \\
\hline 12. & Cyperus compressus $\mathrm{L}$. & Cyperaceae & ++ & FO \\
\hline 13. & C. iria $\mathrm{L}$. & Cyperaceae & +++ & $\mathrm{FO} / \mathrm{M}$ \\
\hline 14. & Diplazium esculentum (Retz.) Sw & Athyriaceae & ++ & $\mathrm{F}$ \\
\hline 15. & Eclipta prostrata (L.) L. & Asteraceae & ++ & $\mathrm{F} / \mathrm{M} / \mathrm{R}$ \\
\hline 16. & Eqisetum diffusum D. Don & Equisetaceae & ++ & M \\
\hline \multirow[t]{2}{*}{17.} & Eragrostis unioloides (Retz.) Nees ex & & & \\
\hline & Steudel & Poaceae & ++ & FO \\
\hline 18. & Gnaphalium polycaulon Pers. & Asteraceae & ++ & - \\
\hline 19. & Grangea maderaspatana (L.) Poiret & Asteraceae & ++ & $\mathrm{C}$ \\
\hline 20. & Hydrocotyle sibthorpioides Lam. & Apiaceae & + & - \\
\hline 21. & Ischaemum rugosum Salisb. & Poaceae & ++ & FO \\
\hline 22. & Kyllinga brevifolia Rottb. & Cyperaceae & +++ & FO \\
\hline 23. & Lindernia antipoda (L.) Alston & Scrophulariaceae & ++ & - \\
\hline 24. & L. ciliata (Colsm.) Pennell & Scrophulariaceae & ++ & $\mathrm{F}$ \\
\hline 25. & L. crustacea (L.) F. Muell. & Scrophulariaceae & ++ & - \\
\hline 26. & Lippia nodiflora (L.) Rich. & Verbenaceae & ++ & $\mathrm{F} / \mathrm{M}$ \\
\hline 27. & Meniscium proliferum (Retz.) Sw & Thelypteridaceae & ++ & - \\
\hline 28. & Mikania micrantha Kunth & Asteraceae & +++ & - \\
\hline 29. & Murdania nudiflora (L.) Brenan & Commelinaceae & ++ & FO \\
\hline 30. & M. vaginata (L.) Brueckns & Commelinaceae & + & FO \\
\hline 31. & Oxystelma esculentum (L. f.) Sm. Hara & Asclepiadaceae & ++ & M \\
\hline 32. & Polygonum. plebeium R. Br. & Polygonaceae & ++ & M \\
\hline 33. & Rorippa benghalensis (DC.) Hara & Brassicaceae & ++ & - \\
\hline 34. & Saccharum spontaneum L. & Poaceae & ++ & FO/GR/M \\
\hline
\end{tabular}


35. Setaria pallidefusca (Schumach.) Stapf \&

C. E. Hubbard

36. Sphaeranthus indicus L.

37. Typha. elephantina Roxb.

\section{Tenagophytees}

1. Acorus calamus L.

2. Aeschynemone asper L.

3. Ammania auriculata Willd.

4. Canna indica L.

5. Ceratopteris thalictroides Brongn

6. Colocasia esculenta (L.) Schott

7. Cyperus corymbosus Rottb.

8. C. difformis L.

9. Echinochloa colona (L.) Link

10. E. stagnina (Retz.) Nees ex Steudel

11. Fimbristylis littoralis Gaud.

12. F. miliacea (L.) Vahl

13. F. schoenoides (Retz.) Vahl

14. Floscopa scandens Lour.

15. Hemarthria compressa (L. f.) R. Br.

16. Hygrophila polysperma (Roxb.) T. Anders

17. Isachne dispar Trin.

18. Leptochloa chinensis (L.) Nees

19. Ludwigia perennis L.

20. Marchantia palmata Nees

21. Mariscus compactus (Retz.) Druce

22. Melochia corchorifolia L.

23. Paspalum scrobiculatum L.

24. Polygonum barbatum (L.) Hara

25. Polygonum hydropiper L.

26. Rotala indica (Willd.) Koehne

27. $R$. rotundifolia (Buch.-Ham. ex Roxb.)

Koehne

28. Rumex dentatus L.

29. Sagittaria trifolia L.

30. Scleria parvula Steudel

31. Smithia ciliata Royle

32. Sphenoclea zeylanica Gaertn.

33. Spilanthes iabadicensis A. H. Moore

34. Veronica anagallis-aquatica $\mathrm{L}$.

35. Vetiveria zizanioides (L.) Nash Hyperhydates

1. Alternanthera philoxeroides Griseb

2. Arundinella bengalensis (Sprengel) Druce

3. A. nepalensis Trin.

4. Brachiaria mutica (Forsskal) Stapf

5. Butomopsis latifolia (D. Don) Kunth

6. Cyperus esculentus L.
Poaceae

Asteraceae

Typhaceae

Araceae

Fabaceae

Lythraceae

Cannaceae

Parkeriaceae

Araceae

Cyperaceae

Cyperaceae

Poaceae

Poaceae

Cyperaceae

Cyperaceae

Cyperaceae

Commelinaceae

Poaceae

Acanthaceae

Poaceae

Poaceae

Onagraceae

Marchantiaceae

Cyperaceae

Sterculiaceae

Poaceae

Polygonaceae

Polygonaceae

Lythraceae

Lythraceae

Polygonaceae

Alismataceae

Cyperaceae

Fabaceae

Sphenocleaceae

Asteraceae

Scrophulariaceae

Poaceae

Amaranthaceae

Poaceae

Poaceae

Poaceae

Butomaceae

Cyperaceae

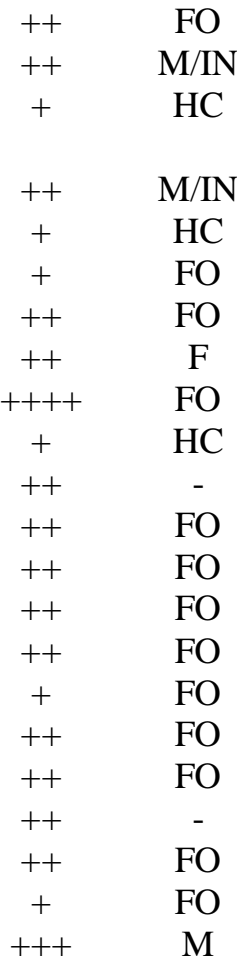

$+\quad$ Soil binder

$++\quad-$

$++\quad-$

$++\mathrm{FO} / \mathrm{M}$

$++\quad \mathrm{M}$

$+++\quad$ FP

$++$

$++$

$+++\quad \mathrm{C}$

$++\quad \mathrm{F}$

$++\quad \mathrm{FO}$

$+\quad \mathrm{FO}$

$++\quad \mathrm{F}$

$++\quad \mathrm{M}$

$++$

$++\quad \mathrm{HC} / \mathrm{FO}$

$++\quad \mathrm{C} / \mathrm{F}$

$++\quad \mathrm{FO}$

$++\quad$ FO

$++\quad \mathrm{FO}$

$++\quad-$

$++\mathrm{HC}$ 
7. C. pilosus Vahl

8. Echinochloa crus-galli (L.) Beauvois

9. Eleocharis acutangula (Roxb.) Schultes

10. E. atropurpurea (Retz.) Kunth

11. Eriocaulon cinereum $\mathrm{R}$. Br.

12. Hydrolea zeylanica (L.) Vahl

13. Hygrophila auriculata (Schumach.) Heine

14. Hymenachne pseudointerrupta C. Mueller

15. Ipomoea carnea Jacq.subsp. fistulosa (Mart. ex Choisy) D. F. Austin

16. Leersia hexandra Swartz.

17. Ludwigia octovalvis (Jacq.) Raven

18. Marsilea minuta L.

19. Monochoria hastata (L.) Solms.

20. M. vaginalis (Brum.) Kunth

21. Oenanthe javanica (Blume) DC.

22. Oryza rufipogon Griff.

23. O. sativa $L$.

24. Panicum paludosum Roxb.

25. P. psilopodium Trin.

26. Paspalidium punctatum (Brum.) A. Camus

27. Paspalum distichum L.

28. Polygonum glabrum Willd.

29. P. lapathifolium L.

30. Ranunculus scleratus L.

31. Rorippa nasturtium-aquaticum (L.) Hayek.

32. Sacciolepis indica (L.) Chase

33. S. interrupta (Willd.) Stapf

34. Schoenoplectus grossus (L. f.) Palla

35. S. juncoides (Roxb.) Palla

36. S. mucronatus (L.) Palla

37. Typha angustifolia $\mathrm{L}$.

\section{Epihydates}

1. Euryle ferox Salisb.

2. Ipomoea aquatica Forssk.

3. Ludwigia adscendens (L.) Hara

4. Nymphaea pubescens Willd.

5. Nymphoides hydrophyllum (Lour.) Kuntze

6. Sagittaria guyanensis Kunth

7. Trapa natans var. bispinosa (Roxb.) Makinno

\section{Vittates}

1. Ceratophyllum demersum $\mathrm{L}$.

2. Chara schweinitzii A. Braun

3. Hydrilla verticillata (L. f.) Royle

4. Potamogeton crispus L.

5. Utricularia aurea Lour.

6. U. exoleta R. Br.

\begin{tabular}{|c|c|c|}
\hline Cyperaceae & + & - \\
\hline Poaceae & ++ & FO \\
\hline Cyperaceae & ++ & FO \\
\hline Cyperaceae & ++ & FO \\
\hline Eriocaulaceae & ++ & FO \\
\hline Hydrophyllaceae & ++ & - \\
\hline Acanthaceae & +++ & M \\
\hline Poaceae & ++ & M \\
\hline Convolvulaceae & +++ & FO \\
\hline Poaceae & ++ & $\mathrm{FW} / \mathrm{H} / \mathrm{IN}$ \\
\hline Onagraceae & ++ & FO/GR \\
\hline Marsileaceae & ++ & - \\
\hline Pontederiaceae & ++ & $\mathrm{F}$ \\
\hline Pontederiaceae & ++ & $\mathrm{C}$ \\
\hline Apiaceae & ++ & M \\
\hline Poaceae & + & $\mathrm{F}$ \\
\hline Poaceae & +++++ & $\mathrm{FO} / \mathrm{GR}$ \\
\hline Poaceae & + & FO \\
\hline Poaceae & ++ & $\mathrm{F} / \mathrm{FO}$ \\
\hline Poaceae & +++ & FO \\
\hline Poaceae & ++ & FO \\
\hline Polygonaceae & ++ & FO \\
\hline Polygonaceae & + & FO \\
\hline Ranunculaceae & ++ & FP \\
\hline Brassicaceae & ++ & FP \\
\hline Poaceae & ++ & M \\
\hline Poaceae & ++ & $\mathrm{F}$ \\
\hline Cyperaceae & ++ & FO \\
\hline Cyperaceae & ++ & $\mathrm{FO}$ \\
\hline Cyperaceae & + & $\mathrm{F} / \mathrm{HC}$ \\
\hline \multirow{2}{*}{ Typhaceae } & ++ & $\mathrm{HC}$ \\
\hline & ++ & $\mathrm{HC}$ \\
\hline Nympheaceae & ++ & $\mathrm{HC}$ \\
\hline Convolvulaceae & ++ & $\mathrm{F} / \mathrm{R}$ \\
\hline Onagraceae & ++ & $\mathrm{F}$ \\
\hline Nympheaceae & ++ & - \\
\hline Gentianaceae & ++ & $\mathrm{F} / \mathrm{R}$ \\
\hline Alismataceae & ++ & $\mathrm{M}$ \\
\hline Trapaceae & ++ & $\mathrm{F}$ \\
\hline Ceratophyllaceae & ++ & $\mathrm{F} / \mathrm{R}$ \\
\hline Characeae & ++ & $\mathrm{M}$ \\
\hline Hydrocharitaceae & ++ & IN \\
\hline Potamogetonaceae & ++ & $\mathrm{C}$ \\
\hline Lentibulariaceae & ++ & M \\
\hline Lentibulariaceae & + & $\mathrm{M}$ \\
\hline
\end{tabular}




\begin{tabular}{|c|c|c|c|c|}
\hline 1. & $\begin{array}{l}\text { Rosulates } \\
\text { Ottelia alismoides (L.) Pers. } \\
\text { Pleustophytes }\end{array}$ & Hydrocharitaceae & ++ & $\mathrm{F} / \mathrm{M}$ \\
\hline 1. & Azolla imbricata (Roxb.) Nakai & Azollaceae & +++ & $\mathrm{C} / \mathrm{FO}$ \\
\hline 2. & Eichhornia crassipes (Mart.) Solms. & Pontederiaceae & ++++ & $\mathrm{C}$ \\
\hline 3. & Lemna minor $\mathrm{L}$. & Lemnaceae & +++ & M \\
\hline 4. & Pistia stratiotes $\mathrm{L}$. & Araceae & +++ & $\mathrm{C} / \mathrm{M}$ \\
\hline 5. & Spirodela polyrhiza (L.) Schleiden & Lemnaceae & +++ & $\mathrm{C}$ \\
\hline
\end{tabular}

\section{Acknowledgements}

We are thankful to the Head, Department of Botany, Post Graduate Campus, T.U. Biratnagar, Nepal for lab facilities. One of the authors $(\mathrm{BN})$ is grateful to the Tribhuvan University for the Ph.D. study leave and the University Grants Commission, Nepal for the research fellowship.

\section{References}

Anonymous (1948 - 1976): Wealth of India: raw materials (I-X), CSIR, New Delhi.

Bala, G. and A. Mukherjee (2007): Useful plants of wetlands in Nadia district, West Bengal. Geobios 34 (4): 253 - 256.

Banerjee, M. L. (1995): Some edible and medicinal plants from east Nepal. J. Bomb. Nat. Hist.Soc. 53: $153-155$.

Cook, C.D.K. (1996): Aquatic and wetland plants of India. Oxford University Press, Oxford-New YorkDelhi.

Dangol, D.R., S.B. Gurung and I. Bhattarai (1986): Lowland rice weeds at the Agronomy Farm, IAAS, Rampur, Chitwan, Nepal. J. Inst. Agric. Anim. Sc. 7: 01-12.

GON (2014): Nepal National Biodiversity Strategy and Action Plan 2014-2020. Government of Nepal, Ministry of Forests and Soil Conservation, Singhadurbar, Kathmandu, Nepal.

GON(2007). Medicinal plants of Nepal. Government of Nepal, Ministry of Forests and Soil Conservation Department Plant Resources, Thapathali, Kathmandu, Nepal.

Hara, H. and L.H. Williams (1979): An enumeration of the flowering plans of Nepal Vol. 2. British Museum (Nat. Hist.), London.

Hara, H., A.O. Chater and L.H. Williams (1982): An enumeration of the flowering plants of Nepal Vol.3. British Museum (Nat. Hist.), London.

Hara, H.W., T. Stearn and L.H. Williams. 1978. An enumeration of the flowering plants of Nepal Vol. 1.British Museum (Nat. Hist.), London.

Hooker, J.D. (1872-1897): The flora of British India, 7 volumes L. Reeve, London.

IUCN/Nepal (1992). Wetlands management programme. A project Document. IUCN/ Nepal.

Misra, R. (1976): Seasonal dynamics and aquatic weeds of the lowlying lands of the mid- Ganga plains. In: Aquatic weeds in South East Asia. (eds.) C.K. Varshney and J. Rzoska, R. W. Junk, Hague. pp. 103-106.

Mitsch, W. J. and J. G. Gosselink (2000): Wetlands. John Wiley and Sons, Inc. New York, Chichester, Weinhelm, Brisbane, Singapore, Toronto.

Niroula, B. and K.L.B. Singh. 2010. Contribution to aquatic macrophytes of Biratnagar and adjoining areas, eastern Nepal. Ecoprint 17: 23-34.

Press, J.R., K.K. Shrestha and D.A. Sutton (2000): Annonated checklist of the flowering plants of Nepal. The Natural History Museum, London.

Siwakoti, M.and S.K. Varma (1999): Plant diversity of eastern Nepal/Flora of plains of eastern Nepal. Bishen Singh Mahendra Pal Singh. Dehra Dun (India). P. 491.

Upadhyay, B.P., S. Jha, U. Koirala and B. Niroula (2011): Inventory of ten auatic habitats of Biratnagar township, Nepal. GEOBIOS 38: 283-288. 